\title{
La gestión del conocimiento: aplicación a la promoción de la salud
}

\author{
Francisco Javier García Marco \\ Área de Biblioteconomía y Documentación \\ Universidad de Zaragoza \\ E-mail: jgarcia@unizar.es
}

\subsection{Resumen}

El campo de la promoción de la salud es examinado como un campo privilegiado para aplicar, probar y promover la filosofía de la gestión del conocimiento. En primer lugar, se analiza el desarrollo de la disciplina, estableciendo el lugar central que ocupa la transferencia del conocimiento en su éxito práctico. En segundo lugar, se consideran los retos y las oportunidades que la sociedad del conocimiento ofrece en dicho campo. En tercer lugar, se presenta un modelo integrado para implementar la gestión del conocimiento, basado en la complementariedad de otros dos modelos: el tradicional del ciclo del conocimiento, y otro multinivel o estratigráfico. Finalmente, se presenta una propuesta práctica de despliegue de la gestión del conocimiento en una administración pública responsable de la promoción de la salud.

Palabras clave: Gestión del conocimiento. Promoción de la salud.

\subsection{Abstract}

Health promotion is examined as a privileged field for applying, testing and promoting the philosophy of knowledge management. First, the development of the discipline is studied, establishing the central role of knowledge transfer for its success. Second, the challenges and opportunities of the knowledge society for health promotion are considered. Third, an integrative model for implementing knowledge management is presented, based in the complementarity of two other models: the cycle of knowledge model and the multilevel or stratigraphic one. Fourth, the importance of a basic ontology in health promotion in a knowledge management strategy is considered. Finally, a practical proposal for a knowledge management implementation in a health promotion public administration is presented.

Keywords: Knowledge Management. Health Promotion. 


\section{Introducción}

Este trabajo explora el campo de la información y documentación en promoción de la salud como un campo privilegiado para la aplicación de una nueva filosofía de la administración: la gestión del conocimiento. Esta nueva perspectiva del liderazgo organizacional cuestiona a los administradores de la promoción de la salud y a los gestores de la información y documentación por igual, y les anima a dar un paso más en sus trabajos al servicio del desarrollo social. No en vano uno de los objetivos clave de la promoción de la salud es precisamente transferir los conocimientos de salud a los agentes responsables y a la población (García Marco, 1999). Esa tarea se enfrenta hoy en día a problemas muy diferentes a los que se daban hace décadas, cuando la información y el conocimiento eran bienes escasos. Actualmente la transferencia del saber de salud se produce en un ambiente caracterizado por el cambio constante, donde la sobrecarga de información, la desinformación y la contrainformación conviven con unas posibilidades inéditas de acceso al conocimiento.

La primera parte del artículo se dedica a estudiar los factores sociales subyacentes al desarrollo disciplinar de la promoción de la salud, hasta llegar al momento actual, en el que asistimos al advenimiento de una nueva organización social - la sociedad del conocimiento - que reclama también un nuevo concepto de la promoción de salud. La sociedad del conocimiento se dibuja como una sociedad globalizada en la que los esfuerzos humanos - el trabajo - se centran cada vez más en las relaciones humanas, la reflexión y la innovación, mientras que la producción de bienes materiales y la gestión de información administrativa se delega de forma también creciente en máquinas. En una sociedad de este tipo, se está viendo cada vez con más claridad que el papel de los responsables públicos y de los directivos no es tanto controlar procesos de producción, como articular, por un lado, los valores y objetivos del cuerpo social y de sus organizaciones, y, por el otro, facilitar y gestionar la creación, transferencia y almacenamiento del conocimiento. Esta realidad se ha concretado desde principios de los años noventa en un movimiento que ha recibido diversas etiquetas: "dirección del conocimiento", "dirección basada en el conocimiento" y, más recientemente, "administración" o "gestión del conocimiento".

La segunda parte del artículo se dedica precisamente a comentar las características fundamentales de esta nueva filosofía de la gestión y a estudiar sus implicaciones para un nuevo concepto de la promoción de la salud.

Finalmente, se realiza una propuesta para el despliegue de la gestión del conocimiento en una administración al servicio de la promoción de la salud. 


\section{La promoción de la salud: de la salud pública al saber de salud}

\subsection{La promoción de salud en el centro del concepto de estado del Bienestar}

La promoción de la salud es uno de los elementos clave del estado social, en cuanto que garantiza de forma propositiva y proactiva el derecho de los ciudadanos a una vida saludable. El contrato social que sustenta la filosofía política que subyace a las modernas democracias establece que el Estado debe ocuparse activamente del bienestar de los ciudadanos. Y ello básicamente de dos formas. Por un lado, complementando la iniciativa privada donde ésta no puede o no está dispuesta a llegar. Por el otro, limitando los daños potenciales que la actividad de los agentes individuales puede producir como resultado de una búsqueda del beneficio que, aunque legítima en principio, puede descuidar los intereses de la comunidad y de sus sectores más desfavorecidos.

Concentrándonos en el campo que nos interesa, no cabe ninguna duda de que en el último siglo se ha producido un gran desarrollo del derecho a la salud y de la tutela ejercida por el Estado. Como resultado de ello, el campo de la promoción de la salud se ha ido ampliando en sucesivos círculos cada vez más amplios hacia una interdisciplinariedad creciente. Este movimiento ha desembocado en la creación de una nueva interdisciplina, donde bajo el liderazgo de la profesión médica se integran saberes y especialistas de numerosos campos de interés. Por otra parte y debido a su importancia política que antes se comentaba, la promoción de la salud se ha situado en la punta de lanza del proceso de integración mundial que se denomina globalización. Vamos a repasar esta fascinante evolución con el objeto de situarnos en el momento actual.

\subsection{De la salud pública a la gestión del conocimiento de salud}

La historia de la promoción de la salud arranca en el concepto de salud pública, centrada en la higiene y, sobre todo, en el control de epidemias. La salud pública es tan vieja como la cultura urbana. Aparece a partir de los esfuerzos de la comunidades urbanas por limitar la expansión de las enfermedades infecciosas - el principal riesgo para la salud hasta principios del siglo XX en la totalidad del mundo - mediante medias de aislamiento - cuarentenas, etc. - y obras públicas - aprovisionamiento y de agua y alcantarillado. El campo de conocimiento que delimitan estas actuaciones técnicas es muy reducido, y se apoya básicamente en dos conceptos: el de "higiene" y el de "peste".

En los umbrales de la época contemporánea la salud pública se convierte en medicina preventiva. Como consecuencia del desarrollo de la ciencia médica, el campo cognitivo de la disciplina se amplía muchísimo. Las tres características más importantes que definen a esta fase son, a nuestro juicio, su relación con el desarrollo de los estados nacionales, su asunción de una teoría científica concreta

Scire. $9: 1$ (jul.-dic. 2003) 151-170. 


\section{Francisco Javier García Marco}

- la teoría de los gérmenes - y la adopción de unos mecanismos de intervención también muy específicos - la vacunación y la educación en una higiene anti-infecciosa. Estos tres elementos configuran, sin duda, una disciplina con características plenamente paradigmáticas. Por otra parte, el énfasis en la higiene como elemento de prevención constituyó el germen de un modelo de intervención que incidía directamente en los estilos de vida de los ciudadanos, más allá de la incidencia puntual que provocaban las campañas puntuales de vacunación. Esta preocupación por los estilos de vida contenía la semilla a partir de la cual podría desarrollarse un nuevo paradigma de corte científico-social.

En la segunda mitad del siglo XX, la segunda fase de la revolución industrial provocó una serie de cambios sociales auténticamente revolucionarios que agrupamos convencionalmente bajo la etiqueta de la "sociedad del consumo". El nuevo modelo social incluía una profunda revolución en la alimentación, las costumbres y estilos de vida con enormes implicaciones para la salud de la población. Se trata de un cambio social y cultural muy profundo, que Alvin Toffler denominó la "segunda ola": el cambio humano más importante desde la invención de la agricultura y de la urbanización. Algunas de las consecuencias más importantes de este cambio social para la salud se han convertido en parte del cara y cruz de nuestra vida cotidiana: la producción industrial masiva a costa del deterioro de nuestro entorno; comodidades físicas impensables años atrás, pero un gran estrés psicológico y social; industrialización de la cadena alimenticia humana a costa de graves riesgos para la salud de poblaciones inmensas, etc.

Como consecuencia de la aparición de nuevos problemas de salud, la reflexión sobre las implicaciones de la nueva situación para la salud pública - cuya configuración y consecuencias resultaban por otra parte evidentes para todos los implicados - recibió un apoyo procedente desde fuera del mundo médico: el aporte crítico de las ciencias sociales y humanas. A partir de investigaciones realizadas en campos ajenos a la medicina tradicional - como la veterinaria, la dietética, la psicología social, la sociología, las ciencias del deporte, la sexología y un largo etcétera - se realizó una crítica de los límites del modo de vida consumista y urbano. El área de la prevención de enfermedades se alimentó de todo este aporte y se configuró como un campo interdisciplinar lleno de especialidades muy boyantes, donde, por otra parte, empezaban a plantearse graves problemas de organización y gestión del conocimiento.

Actualmente el campo de la promoción de la salud, especialmente si aceptamos la noción de bienestar explícita en la definición de la Organización Mundial de la Salud, es un campo interdisciplinar; es decir, integra los saberes de disciplinas muy diversas. Más aún, se alimenta de la interacción de teóricos, investigadores y profesionales de distintas disciplinas. Así, por ejemplo, psicólogos sociales y médicos contribuyen conjuntamente al campo de la sexología; o sociólogos,

Scire. $9: 1$ (jul.-dic. 2003) 151-170. 
antropólogos, veterinarios y médicos lo hacen en el ámbito de la alimentación sana. Si se pudiera sobrevolar virtualmente el campo de la promoción de la salud a principios del siglo XXI nos asombraría la importancia creciente de esos recorridos transversales - transdisciplinares - a lo largo de muchas disciplinas que aparecen cuando se consideran temas que afectan a toda la sociedad, como los que ya hemos mencionado de la alimentación, el medio ambiente, la sexualidad o la actividad física.

Por si todos estos cambios no fueran en sí mismos suficientemente revolucionarios, a finales de los años ochenta y principios de los noventa la revolución informática se aceleró a raíz del estallido de la WWW. Como consecuencia empieza a nacer un nuevo entorno social cuyas implicaciones apenas comenzamos a comprender. Todo indica que en el momento actual nos encontramos en el inicio de una nueva etapa de la evolución de la humanidad, a la que cada vez más se designa con el nombre de "sociedad del conocimiento". En este ambiente de grandes esperanzas y preocupaciones la promoción de la salud se ha configurado ya como un campo transdisciplinar en ebullición cuyas ambiciones y dinámica entran plenamente dentro de esa nueva configuración social emergente, un sistema social en permanente mejora gracias al énfasis y recursos dedicados a la creación y transferencia más rápida, generalizada y eficaz de conocimientos. Conocimientos que consisten, en definitiva, en datos sobre la realidad y de modelos para interpretarlos y actuar a partir de ellos.

\subsection{Oportunidades de la sociedad del conocimiento para la promoción de la salud}

El elemento más evidente de la nueva etapa es el extraordinario desarrollo de las tecnologías de la información y la amplitud de su calado en todos los ámbitos de actividad de las sociedades desarrolladas. Las tecnologías de la información han facilitado el control automático de las máquinas de la segunda revolución industrial, liberando todavía más mano de obra de los sectores primario y secundario. Además, la automatización está invadiendo el trabajo administrativo - ejecutado cada vez más por agentes informáticos - con unas consecuencias que todavía resultan difíciles de prever, pero cuya tendencia general resulta previsible. Como consecuencia, la fuerza de trabajo de las sociedades desarrolladas se ha ido volcando hacia los servicios, destacando entre ellos en la actualidad aquellos que descansan en el uso intensivo de conocimientos y en la importancia de las relaciones personales, como la salud, el ocio, la educación, la investigación, la gestión y las ventas.

La promoción de la salud - una actividad que consiste básicamente en transferir resultados de investigación a los agentes sociales y en gestionar esos procesos - se está beneficiando extraordinariamente de esta situación por muchas 
razones. En primer lugar, se trata de una actividad que satisface deseos y derechos vitales del común de los ciudadanos. En segundo lugar, puede ser rentable económicamente en la medida en que puede ahorrar gastos posteriores. En tercer lugar, al descansar en conocimientos y procesos de comunicación - pues es una actividad cuya materia prima es sin duda la información-, es susceptible de beneficiarse enormemente de los avances informáticos y telemáticos. Finalmente, en cuanto a ocupación perteneciente al sector de los "nuevos servicios", puede esperar la ampliación de las personas que la practican.

\subsection{Retos de la sociedad del conocimiento a la promoción de la salud}

Ahora bien, la nueva configuración socio-tecnológica plantea también importantes retos a los administradores de la promoción de la salud. Merece la pena tratar con algo más de profundidad seis de ellos.

1. La integración de conocimientos en un entorno globalizado. A largo plazo, la extraordinaria y perjudicial - en cuanto excesiva - redundancia de nuestros sistemas de memoria y comunicación tiene que dar paso a servicios de información y bibliotecas digitales globales que condensarán toda la información disponible sobre sus dominios en tiempo real y de forma totalmente integrada, constituyendo una suerte de "cerebros mundiales" (Matheson, 1995). El proyecto del genoma humano ha mostrado los potenciales que ofrece la integración de los procesos de documentación, información y comunicación dentro de sistemas más amplios de gestión del conocimiento, un nuevo concepto que analizaremos con más detalle en la segunda parte de este trabajo.

2. La dinámica especialización-interdisciplinariedad. La sociedad-red característica de la "tercera ola", por otra parte, potencia aun más la dinámica especialización-interdisciplinariedad en toda la sociedad, y, por supuesto, en el campo de la promoción de la salud. Los conocimientos disponibles son mayores, pero también exigen una actividad profesional de gestión de los mismos para facilitar su almacenamiento y difusión por todo el cuerpo de usuarios potenciales.

3. El filtrado y modulado de la información. Los problemas de promoción de la salud no se pueden tratar de forma general para toda la sociedad, sino que se deben ajustar según la población objeto y los escenarios en los que se realizan. No es lo mismo, por ejemplo, tratar el tema de la prevención del SIDA entre adolescentes, que entre personas en reclusión o turistas. Solo una adecuada modulación de la información resolverá los problemas de inadecuación y saturación de información tan característicos de nuestros tiempos. 
4. El conocimiento de calidad. El enunciado de misión implícito de la sociedad del conocimiento se orienta hacia la consecución de representaciones de la realidad progresivamente más verídicas, exactas, profundas, intersubjetivas, integradas entre sí y aceptadas por la sociedad. Eso no es posible sin integrar el ciclo problema-información-reflexión-acción junto con la extraordinaria base tecnológica a nuestra disposición (1998).

5. El aprendizaje continuo. Otra característica significativa de la "sociedad del conocimiento" es que el conocimiento se sitúa en el centro de los criterios de legitimidad del poder social, desplazando aun más a otros elementos del sistema social de autoridad como la tradición. El experto ya no es creado por un "título", sino que esa condición se gana día a día mediante la formación permanente. Como resultado del imparable proceso de cambio, el aprendizaje continuo ya no es un desideratum, sino una tarea ineludible cuya responsabilidad debe ser asumida por los que ejercen el liderazgo en las instituciones donde se promociona la salud (Nutley, 2001).

6. La gestión de las redes de conocimiento. Los agentes de promoción de salud, especialmente los que tienen responsabilidades de dirección y coordinación, son cada vez más conscientes de que han dejado de ser los depositarios únicos del conocimiento de promoción de salud y que su tarea consiste cada vez más en convertirse en expertos en gestionar las complejas tramas de intercambio de conocimientos que, a su vez, transfieren los conocimientos sobre la salud a la población y a los grupos que la componen.

En definitiva, el reto del experto en promoción de la salud es cada vez más custodiar y comunicar conocimientos de calidad, diversos - en cuanto que proceden de disciplinas, escenarios, etc., distintos-, pero integrados, dirigidos a receptores específicos, en entornos de aprendizaje e innovación continuos, con una estructura compleja -múltiples nodos y niveles-, y gestionados por expertos.

Para ello, es necesario acometer conscientemente un trabajo que hasta ahora se hacía más bien de forma intuitiva, y para el cual pueden resultar extraordinariamente útiles los conceptos de una disciplina $-\mathrm{o}$, si se quiere, de un nuevo paradigma - de la gestión que ha emergido a lo largo de los años noventa, a saber, la gestión o administración del conocimiento, que se trata a continuación.

\section{La gestión del conocimiento en promoción de salud}

\section{1. ¿Qué es la gestión del conocimiento?}

La gestión del conocimiento es un campo de investigación y acción de carácter interdisciplinar que surgió a partir de la integración de conocimientos procedentes de la psicología, la sociología, la administración de empresas, la economía,

Scire. $9: 1$ (jul.-dic. 2003) 151-170. 
la informática y las ciencias de la documentación, entre otras disciplinas, en torno al problema de la administración del conocimiento en las organizaciones.

La gestión del conocimiento es una filosofía de la administración de organizaciones que nace de la percepción de la íntima conexión y de las enormes posibilidades de sinergia que existen entre funciones organizacionales tan diversas como la gestión de la comunicación interna y externa, la documentación, la gestión de las tecnologías de la información y de la comunicación, la formación - requerida por el empleado tanto como por la empresa - y la asesoría - incluyendo la asistencia interna entre expertos - . La integración de todos estos aspectos se percibe como un elemento clave de la planificación estratégica y de la función de liderazgo, que, a su vez, se concibe más como una instancia de clarificación de valores, de facilitación y catálisis de iniciativas, que como una instancia de control, misión que se delega más bien a la función de administración.

Los procesos de integración de funciones como el aprendizaje y la documentación no son extraños ni nuevos en sí mismos. Un ejemplo puede ser la colaboración de los bibliotecarios en la formación del personal de atención sanitaria (Layton \& Hahn, 1995) o, en dirección inversa, la participación de los técnicos sanitarios en la formación de las colecciones y desarrollo de los servicios de la biblioteca digital. Sin embargo, la filosofía de la gestión del conocimiento pretende que dicha integración se realice de forma consciente y sistemática.

Por otra parte, el concepto de administración del conocimiento incorpora como elementos clave tanto un nuevo diagnóstico del entorno como una nueva filosofía de la organización. El entorno se diagnostica tanto a partir del impacto de la revolución telemática y la sociedad de la información, como por el futuro advenimiento de una sociedad basada en el conocimiento y en un nuevo concepto del trabajo, del ocio y de la ciudadanía. Las nuevas filosofías del desarrollo organizacional que forman parte del núcleo conceptual de la gestión del conocimiento son las que han cristalizado en torno a los conceptos de organización que aprende (learning organization), en red (networking) y horizontal.

Finalmente, la gestión del conocimiento aparece como concepto clave para asegurar la competitividad en una sociedad en la que existe fácil acceso a los mercados de capital, trabajo, productos y servicios. Efectivamente, la abundancia de los recursos disponibles en el mercado hace que las empresas dependan menos de los recursos tradicionales - humanos, materiales y financieros - y que su ventaja competitiva proceda sobre todo por la idiosincrasia de su comprensión de las demandas y ofertas existentes en la sociedad y de sus planes para aprovechar esa comprensión de forma pertinente e innovadora. En definitiva, la información asimilada, el conocimiento, se convierte en la ventaja competitiva clave de cualquier organización - pública o privada - en el nuevo entorno social.

Scire. $9: 1$ (jul.-dic. 2003) 151-170. 


\subsection{Gestión del conocimiento v. gestión de la información}

Ahora bien, el conocimiento va mucho más allá de la información. El conocimiento no es solo la información exterior captada por una persona o una institución en su conjunto. Es también el conjunto de esquemas - algunos aprendidos, otros mejorados o inventados completamente - con los que las personas y las organizaciones interpretan esa información. A su vez dichos esquemas definen sus formas de actuar y de transformar, recíprocamente, esa realidad que les informa desde fuera. Por eso, la gestión del conocimiento no se puede confundir con la mera gestión de información y documentación, aunque sin duda la integra. La administración del conocimiento exige que el proceso de información y documentación se inserte realmente dentro de los procesos de creación y utilización de conocimiento de las personas y de sus organizaciones. La gestión del conocimiento abarca, por tanto, un dominio mayor que la gestión de la información. Sin embargo, la gestión de la información ocupa un lugar clave dentro del proceso de gestión del conocimiento, pues asegura que el conocimiento capturado se almacene, administre y difunda adecuadamente. Por otro lado, la gestión del conocimiento exige del profesional de la información y la documentación que no se centre tanto en el documento como fin en sí mismo, sino que lo considere un medio para codificar, almacenar y difundir el conocimiento relevante; sin descuidar, claro está, de forma secundaria, su función de conservador de la memoria organizacional.

Desde el punto de vista del documentalista destaca la incorporación de un enfoque global del problema de la información en todos sus aspectos: la captación (antenas y observatorios), la normalización y procesamiento, la gestión y almacenamiento, la difusión muticanal - atendiendo tanto a los medios tecnológicos como a la interacción humana y social - y el filtrado.

\subsection{La gestión del conocimiento en el mundo de la salud}

La literatura sobre la aplicación de la gestión del conocimiento a las ciencias de la salud es muy amplia. Tras realizar en febrero del 2001 una búsqueda en el Social Science Citation Index de la expresión knowledge management, las ciencias de la salud aparecieron como una de las áreas aplicativas más importantes, con casi el cinco por ciento de las referencias localizadas (febrero del 2001). Pero no se trata tan solo de atención por parte de los académicos, antes al contrario. La gestión del conocimiento como práctica está muy avanzada en el campo de la salud. Un ejemplo señero lo constituye el Human Genome Project (Boguski, 1995) (2).

Tras intentar clarificar que la administración del conocimiento y las ciencias de la salud configuran imbricados en una colaboración creciente, es el momento de analizar con más detalle qué es la "gestión del conocimiento" y qué aporta de nuevo.

Scire. $9: 1$ (jul.-dic. 2003) 151-170. 


\subsection{El ciclo y los niveles de la gestión del conocimiento}

La teoría de la administración del conocimiento se sustenta en dos modelos principales: un modelo dinámico - el ciclo del conocimiento - y otro estructural - los niveles de la administración del conocimiento (García Marco, en edición). Vamos a repasar someramente sus elementos principales. El ciclo del conocimiento contempla cuatro grandes fases: la creación de conocimiento, la publicación o difusión primaria, la memoria - almacenamiento, ordenación y recuperación- y el aprendizaje o adquisición. Ninguna de estas fases se percibe de forma aislada, sino que, por el contrario, se consideran funciones fuertemente ensambladas.

En realidad, como ya sabemos, la integración entre las fases del ciclo del conocimiento existe de forma natural en la actividad normal de las organizaciones - como no podría ser de otra manera-, pero la experiencia demuestra que suele sufrir muchos problemas e ineficiencias. Por su parte, cualquier administración en cuanto que sistema de procesamiento de información y conocimiento desarrolla estas funciones en mayor o menor grado. El problema de cualquier organización no es tanto implementar dichas funciones - aunque pueda serlo en entidades que están comenzando su andadura - sino más bien sistematizar,

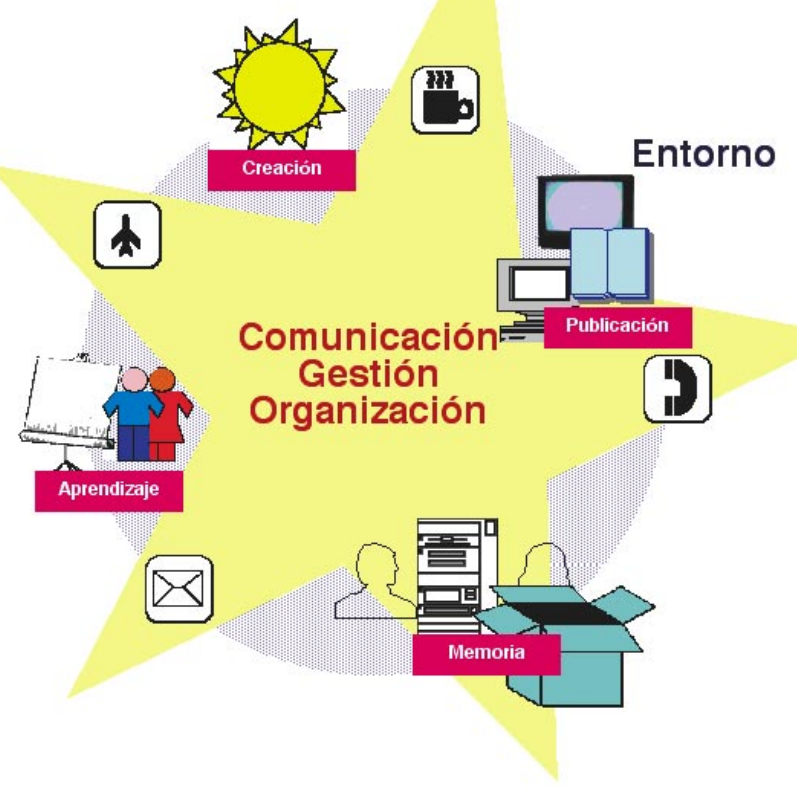

Figura 1. El ciclo del conocimiento

Scire. $9: 1$ (jul.-dic. 2003) 151-170. 
modelar, integrar y optimizar el sistema real existente. Por ello es necesario procurar una fuerte conexión entre todas las fases del ciclo del conocimiento (figura 1) a través de a) una actividad dedicada e inteligente, de un liderazgo efectivo, que ha de actuar a través de unas modalidades y canales de comunicación muy diversos - contactos interpersonales, conferencias, seminarios, tecnologías de comunicación, etc. - ; b) un plan de gestión del ciclo del conocimiento; y c) una organización intelectual del dominio de acción de la institución y de sus recursos que se concreta en una ontología corporativa o científica.

El modelo diacrónico del ciclo de conocimiento se complementa con un modelo estratigráfico (figura 2) que aporta los diferentes aspectos a considerar a lo largo de todo el proceso. Lo más importante del gráfico anterior (García Marco, en edición) - en cuyo detalle no podemos entrar en el espacio de este trabajo- es tomar nota de la importancia de una consideración global de los múltiples factores que inciden en la actividad de administrar el conocimiento.

Los responsables conceden, por lo común, gran importancia al factor tecnológico. En este nivel de actuación los retos se concentran en seis grandes áreas de aplicación: las bases de datos de referencia, la información contextual, los repositorios de datos clínicos, los sistemas de apoyo a la dirección, y los sistemas interactivos de comunicación e información basados en la Internet (Rodrigues, 2000).

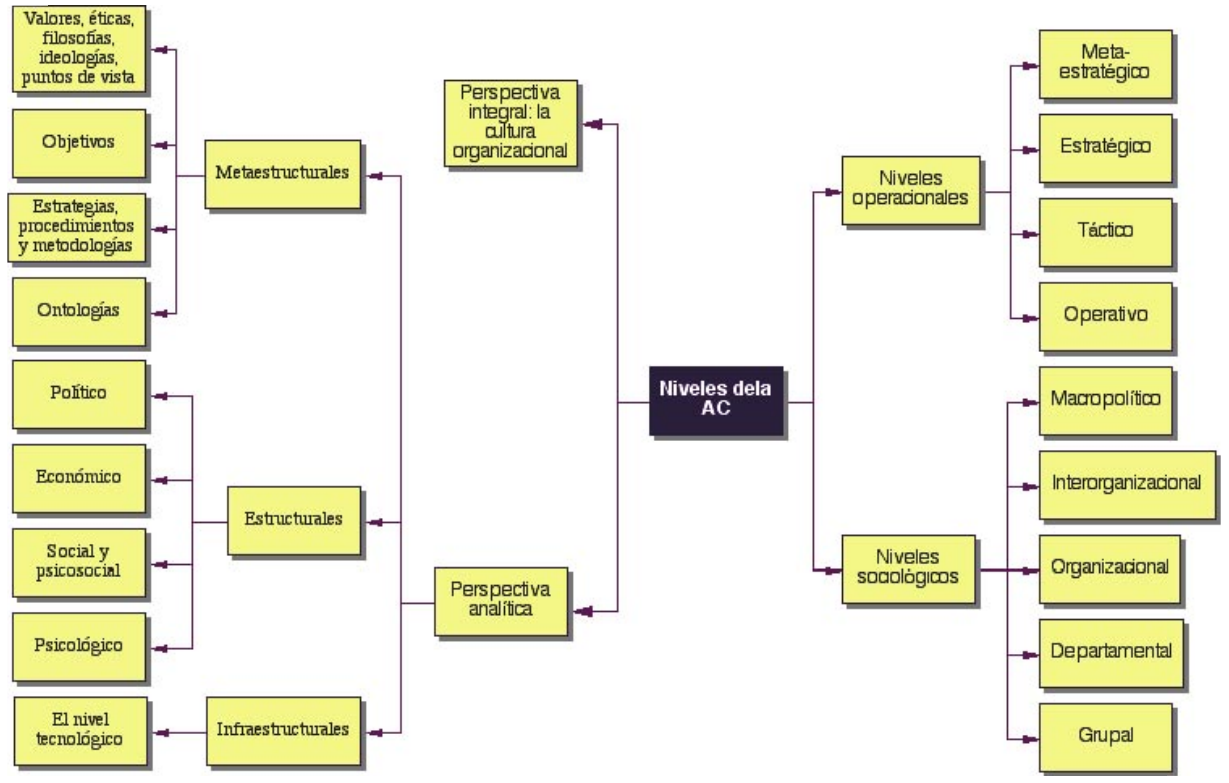

Figura 2. Los niveles de la gestión del conocimiento

Scire. $9: 1$ (jul.-dic. 2003) 151-170. 
El factor tecnológico constituye, sin duda, el signo de los tiempos. Sin embargo, conviene insistir en que la literatura científica está llena de estudios que muestran casos prácticos de gestión errónea por centrarse demasiado en uno de ellos en detrimento de los demás. Un defecto muy común es centrarse en la infraestructura tecnológica o en los problemas financieros, en perjuicio de factores que resultan incluso más importantes como la cultura organizacional (3) o la clarificación conceptual del dominio de actuación de la organización, lo que actualmente se conoce como la "ontología organizacional". Veamos este factor crítico con algo más de detalle, debido a su importancia para construir el mapa del territorio de intervención y colaboración.

\subsection{Hacia una ontología del saber de salud}

La ontología del campo de trabajo constituye un marco para integrar conocimientos, repartir tareas y, en definitiva, construir una comunidad que comparte conocimientos de forma eficaz. La ontología de la promoción de la salud constituye el mapa que determinan cuales son los elementos del "saber de salud" - los conocimientos sobre la salud - y cual es la relación entre ellos. Bajo el patrocinio y dirección de la Unión Europea se ha realizado durante la pasada década un importante trabajo en la clarificación del mundo conceptual de la promoción de la salud, que ha resultado en la confección de un tesauro en once lenguas europeas (Febrel et al., 1997). A partir de un trabajo de reflexión sobre los resultados de esa experiencia, podemos determinar que la ontología básica de la promoción de la salud se puede organizar en nueve grandes clases (figura 3):

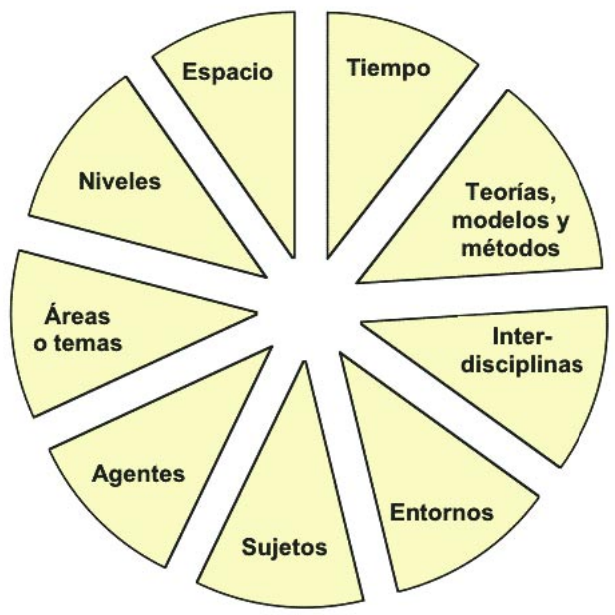

Figura 3. Clases de conocimiento en el saber de salud

- Un cuerpo disciplinar propio compuesto por teorías, modelos, métodos y políticas específicas.

- Un conjunto de conocimientos interdisciplinares relevantes procedentes de otras materias de carácter básico o auxiliar, como la Psicología Social, la Administración, etc. 
- Unos agentes de promoción de salud: políticos, gerentes, médicos, maestros, trabajadores sociales, educadores familiares, etc.

- Unos destinatarios o sujetos de las políticas de promoción de salud: niños, adolescentes, jóvenes, trabajadores, etc.

- Unos escenarios o entornos específicos donde se desarrolla la promoción de la salud: escuelas, cárceles, barrios, etc.

- Unos temas transversales básicos - las áreas de aplicación- que abarcan la salud de los diferentes sistemas funcionales humanos - incluyendo los aspectos psicológicos y relacionales - , sus trastornos y los comportamientos ligados con ellos.

- Unos niveles de intervención: políticos, sociales, grupales, relacionales, psicológicos, fisiológicos, tecnológicos, etc.

- La posible contextualización espacial por países, regiones, localidades, etc.

- La posible contextualización cronológica. Por ejemplo, modelos de intervención que han pasado a la historia o posibilidades prospectivas.

\section{Despliegue de la administración del conocimiento en una administración de la salud}

\subsection{El modelo de intervención}

Un problema típico de la implantación de la gestión del conocimiento en cualquier organización es la magnitud y complejidad de la tarea, dado que afecta a funciones transversales que implican a la totalidad de la organización. A diferencia de actividades específicas como la mejora de la eficiencia financiera o la implantación de un plan de marketing, la gestión del conocimiento se parece mucho al despliegue y mantenimiento de la gestión de calidad. Como la calidad, la información y el conocimiento imbrican - por presencia o ausencia-toda la vida y actividades de la organización y afectan a todos y cada uno de sus miembros y unidades, y exigen el compromiso de todos ellos, empezando, por supuesto y de manera muy especial, por la alta dirección.

Dada la amplitud y complejidad de la tarea recomendamos un abordaje en dos niveles de actuación diferentes, que se concretarán en sendos programas (figura 4). Por un lado, un programa de gestión del conocimiento en la organización, que consistirá, en primer lugar, en la auditoría del estado actual de las estructuras y procesos de gestión del conocimiento y, en segundo lugar, de la elaboración e implementación del plan de intervención subsiguiente. Por el otro, un programa de proyectos piloto, esto es, la puesta en marcha y consolidación de experiencias de innovación en la gestión de la información y del conocimiento que funcionen como ejemplares compartidos y como test de relevancia y eficiencia de los planes

Scire. $9: 1$ (jul.-dic. 2003) 151-170. 


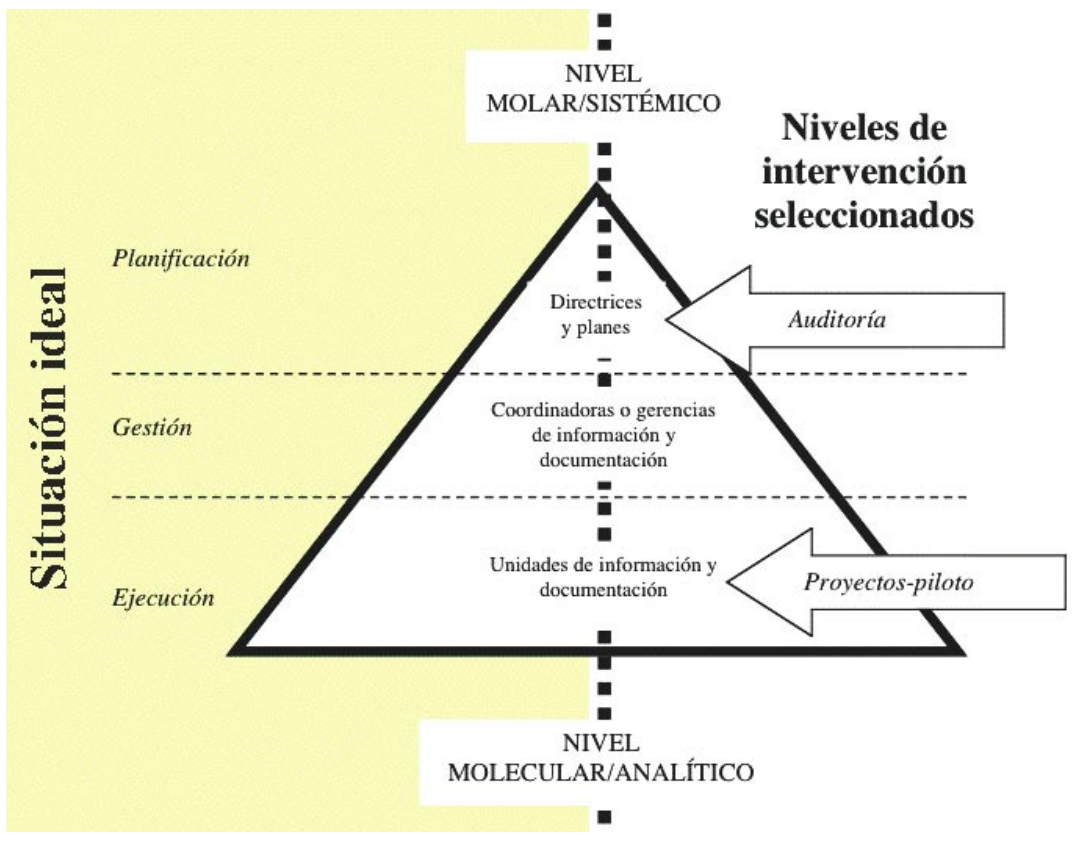

Fig. 4. Modelo de intervención

de alto nivel. Se trata de promover "comunidades de práctica" que sirvan como puntos de experimentación y también, en la medida en que acumulan éxitos basados en la experiencia concreta, en puntos de referencia. Al final del proceso y en como situación extremadamente ideal, cada unidad de la organización debería constituir un equipo de gestión del conocimiento. Así pues, el modelo contempla la intervención en dos niveles: un nivel molar - la planificación global del sistema - , y un nivel molecular y analítico - funcionamiento de las unidades de información-.

Aunque tiene sus inconvenientes, la adopción de un modelo de "dos velocidades" tiene muchas ventajas prácticas. En primer lugar, a través de la retroalimentación entre ambos niveles se asegura una dinámica de investigación-acción, de interacción entre teoría y práctica y de conjunción entre coordinación central y diversidad. En segundo lugar, se evita tanto el riesgo de una planificación centralizada que retrasaría la adopción de medidas y la consecución de experiencia práctica, como el de la atomización de esfuerzos, la dispersión de recursos y los resultados incompatibles que produce la alternativa opuesta. En definitiva, la combinación de una estrategia "de arriba hacia abajo" con otra "de abajo hacia arriba" asegura una gestión sistémica del proceso a la par que una adecuada "infección" 
de la organización. La experiencia de la gestión del conocimiento nos indica que muchas veces existen grupos en las organizaciones que gestionan el conocimiento de forma muy eficaz aunque no existan políticas corporativas en ese sentido (a veces denominadas comunidades de práctica en el lenguaje técnico de la gestión del conocimiento). Una función importante de una dirección que se decide a planificar e implementar un plan de gestión del conocimiento es recoger esas iniciativas, reconocerlas, apoyarlas y utilizarlas de trampolín de todo el proceso.

Desde el punto de vista dinámico y diacrónico, el proceso se conceptualiza en un modelo secuencial bianual compuesto de tres grandes fases: auditoría, planificación e intervención.

\subsection{Objetivos del plan despliegue y resultado esperados}

Además de modelar el proceso de intervención en sus grandes rasgos, es necesario establecer de la forma más clara posible los objetivos - resultados deseables - y los mecanismos del plan de despliegue de la gestión del conocimiento en nuestra organización. Algunos de los objetivos típicos del plan de intervención son los siguientes:

- Iniciar y consolidar una estructura cíclica bianual de mejora continua de la gestión del conocimiento en la organización.

- Iniciar un proceso de reflexión colectiva que incorpore a la cultura organizacional de la organización el concepto del valor de la información y del conocimiento como activo de una importancia semejante a los recursos humanos, materiales o financieros, y de la necesidad de su gestión profesional y científica.

- Analizar las estructuras y procesos de información en la organización.

- Propuesta de un plan de mejora de dichas estructuras y procesos para toda la organización a largo, medio y corto plazo.

- Interconectar a todos los profesionales y funcionarios relevantes para la promoción de la gestión de la información y el conocimiento en el campo de acción de la organización.

- Inventariar experiencias relevantes de otras administraciones locales, autonómicas, nacionales e internacionales; e iniciar con ellas colaboraciones y alianzas.

- Crear una estructura de gestión que dirija e impulse los procesos anteriores.

Entre los resultados finales esperados del proceso de despliegue de la administración del conocimiento deberían estar los siguientes:

- La creación de una estructura organizativa de mejora permanente de la calidad en gestión de la información y conocimiento. 
- La clarificación del mapa de recursos y flujos de información y documentación (deseado y real).

- La creación de un plan de optimización de los subsistemas de información, documentación, publicación y aprendizaje.

- La constitución de una red de unidades de gestión del conocimiento que integre los procesos de creación, publicación, documentación y aprendizaje organizacional en los distintos niveles administrativos.

- La adopción de un plan de infraestructuras telemáticas, informáticas y de otro tipo que resuelvan las necesidades infraestructurales de la red de gestión del conocimiento.

\subsection{Las grandes fases del plan de despliegue}

Como en el caso de la calidad total y en general de cualquier proceso semejante, las fases naturales a considerar son la auditoría o análisis de la realidad, la planificación o previsión, la implantación o ejecución, la evaluación o revisión, y la mejora continua.

Una vez secuencializadas, las fases de despliegue de la gestión del conocimiento podrían distribuirse temporalmente de la siguiente manera, bien entendido que realizamos la propuesta a modo de ejemplo:

1. Diseño e implementación del plan de acción y de la estructura organizativa (primer semestre del año 1)

2. Auditoría de los recursos (estructura) y flujos (dinámica) de información, conocimiento y documentación de la organización y su entorno, y recogida de ideas para un plan de mejora (segundo semestre del año 1).

3. Realización de un encuentro de profesionales y funcionarios interesados en la gestión de la información y el conocimiento en el campo de especialización para ampliar la lluvia de ideas e iniciar la discusión generalizada (primavera del año 1).

4. Elaboración del plan de intervención y mejora (segundo semestre del año 2)

5. Evaluación del proceso (finales del año 2)

6. Reinicio del ciclo de mejora (año 3)

\subsection{La estructura organizativa del plan de intervención}

Por supuesto, la estructura concreta dependerá de las condiciones de la entidad, de su tamaño, del grado de implicación de su dirección y trabajadores, etc. La propuesta que presentamos a modo de ejemplo en la tabla I consta de siete niveles. 


\begin{tabular}{|c|c|c|c|c|}
\hline Equipo & Funciones & Componentes & Creación & Reuniones \\
\hline $\begin{array}{l}\text { Responsables } \\
\text { institucionales }\end{array}$ & $\begin{array}{l}\text { Dirección política. Comunicación ins- } \\
\text { titucional. } \\
\text { Representación pública. } \\
\text { Sanción formal del proyecto. } \\
\text { Aprobación, asunción y sanción formal } \\
\text { de las políticas }\end{array}$ & $\begin{array}{l}\text { Responsable político } \\
\text { y directores generales }\end{array}$ & Inmediata & Ordinarias \\
\hline $\begin{array}{l}\text { Estudio y } \\
\text { animación }\end{array}$ & $\begin{array}{l}\text { Funciona como secretaría permanente } \\
\text { del equipo de auditoría y planificación. } \\
\text { Prepara la agenda, materiales y recur- } \\
\text { sos necesarios para el trabajo de las } \\
\text { demás instancias. } \\
\text { Determina las acciones de motivación } \\
\text { y formación necesarias para asegurar } \\
\text { el éxito del proyecto. } \\
\text { Trabajan con una metodología que } \\
\text { muestra a los demás grupos como } \\
\text { proceder } \\
\text { Realizan el seguimiento de los proyec- } \\
\text { tos pilotos }\end{array}$ & $\begin{array}{l}\text { Cinco personas muy } \\
\text { comprometidas con el proyecto }\end{array}$ & 3 meses & $\begin{array}{l}\text { Cada dos } \\
\text { semanas }\end{array}$ \\
\hline $\begin{array}{l}\text { Auditoría y } \\
\text { planificación }\end{array}$ & $\begin{array}{l}\text { Dirige y aprueba el trabajo del equipo } \\
\text { de estudio y animación. } \\
\text { Proporciona y solicita información al } \\
\text { equipo de estudio y animación } \\
\text { Coordina las acciones de las unidades } \\
\text { administrativas más importantes para } \\
\text { el éxito del proyecto. } \\
\text { Dirige y aprueba el inventario de } \\
\text { recursos } \\
\text { Dirige y aprueba el análisis de flujos } \\
\text { Propone a los responsables institucio- } \\
\text { nales políticas y procedimientos a esta- } \\
\text { blecer (v.g. archivo, automatización de } \\
\text { bibliotecas, servicios telemáticos, etc.) }\end{array}$ & $\begin{array}{l}\text { Responsables de: } \\
\text { Formación } \\
\text { Publicaciones } \\
\text { Investigación y desarrollo } \\
\text { Informática y Telemática } \\
\text { Información estadística } \\
\text { Información legislativa } \\
\text { Información administrativa } \\
\text { (comunicación interna) } \\
\text { Información bibliográfica } \\
\text { Archivo } \\
\text { Comunicación externa } \\
\text { Otros expertos relevantes } \\
\text { Proyectos piloto }\end{array}$ & 6 meses & $\begin{array}{l}\text { Cada dos } \\
\text { meses }\end{array}$ \\
\hline $\begin{array}{l}\text { Coordinadora } \\
\text { de círculos de } \\
\text { calidad }\end{array}$ & $\begin{array}{l}\text { Compartir experiencias y abstraer su } \\
\text { validez de cara a la mejora del plan de } \\
\text { intervención }\end{array}$ & $\begin{array}{l}\text { Un representante de cada } \\
\text { círculo de calidad }\end{array}$ & 3 años & Cada año \\
\hline $\begin{array}{l}\text { Asamblea de } \\
\text { círculos de } \\
\text { calidad }\end{array}$ & $\begin{array}{l}\text { Reforzamiento social de la cultura de } \\
\text { la organización en lo referente a la } \\
\text { gestión de la información y el conoci- } \\
\text { miento por medio de la celebración y el } \\
\text { intercambio de experiencias. } \\
\text { Tomará la forma de un congreso. }\end{array}$ & $\begin{array}{l}\text { Gestores, profesionales y usua- } \\
\text { rios que componen los círculos } \\
\text { de calidad }\end{array}$ & $\begin{array}{l}3 \text { años y } \\
\text { medio }\end{array}$ & $\begin{array}{l}\text { Cada dos } \\
\text { años }\end{array}$ \\
\hline $\begin{array}{l}\text { Círculos de } \\
\text { calidad } \\
2 \text { niveles: } \\
\text { - sección } \\
\text { - servicio }\end{array}$ & $\begin{array}{l}\text { Realizan el análisis estratégico de las } \\
\text { directrices de calidad en gestión de la } \\
\text { información y el conocimiento en su } \\
\text { servicio. } \\
\text { Promueven, planifican e implantan } \\
\text { las directrices de calidad en su nivel } \\
\text { respectivo. } \\
\text { Evalúan el funcionamiento. }\end{array}$ & $\begin{array}{l}\text { Equipos que integran los res- } \\
\text { ponsables y profesionales de las } \\
\text { secciones y servicios, así como } \\
\text { una representación de los } \\
\text { usuarios y proveedores } \\
\text { Se podría considerar la posibi- } \\
\text { lidad de incorporar círculos de } \\
\text { calidad de asociaciones y } \\
\text { colectivos externos. }\end{array}$ & 2 años & $\begin{array}{l}\text { Cada dos } \\
\text { meses }\end{array}$ \\
\hline $\begin{array}{l}\text { Equipo de } \\
\text { investigación }\end{array}$ & $\begin{array}{l}\text { Investiga sobre paradigmas, teorías, } \\
\text { metodologías y líneas de trabajo en } \\
\text { información y documentación admi- } \\
\text { nistrativa y ciudadana en intervención } \\
\text { social para la salud } \\
\text { Apoya científicamente la labor política } \\
\text { y de gestión }\end{array}$ & $\begin{array}{l}\text { Investigadores de la Universidad } \\
\text { Becarios de investigación }\end{array}$ & 6 meses & $\begin{array}{l}\text { Cada } \\
\text { semana }\end{array}$ \\
\hline
\end{tabular}




\section{Conclusiones}

La promoción de la salud se apoya de forma muy importante en la transferencia de conocimientos a la población y a los agentes promotores. Esos conocimientos no han dejado de ampliarse como consecuencia de una concepción más abierta de lo que es la salud, y de las aportaciones de las ciencias de la salud y de las ciencias sociales y humanas al objetivo del bienestar físico, psicológico y social de los seres humanos.

Aunque este crecimiento supone una ventaja indudable, también plantea muchos problemas de absorción y mantenimiento dentro del inmenso campo del saber de salud. Por ello, es necesario abordar profesionalmente el problema de gestionar ese proceso de transferencia de conocimientos. Para ello, la filosofía de la administración del conocimiento nos puede servir de marco teórico y práctico con el que integrar y gestionar las fases del ciclo del conocimiento - creación, publicación, memoria y aprendizaje - y los diferentes niveles que inciden en él -ético, ontológico, político, social, económico, psicosocial, psicológico y tecnológico- . Entre todos ellos, destaca la importancia de elaborar un mapa del territorio del "saber de salud", de la ontología disciplinar de la promoción de la salud.

El despligue de la gestión del conocimiento en la organización de promoción de salud es un problema de índole práctica de gran importancia. En este trabajo proponemos un plan inicial de carácter bianual que combina la actuación a nivel molar - un plan conjunto para toda la organización - y a partir de comunidades de práctica y proyectos piloto - a nivel "molecular"-. Como en otros procesos de despliegue, se habrá de partir de la auditoría, para proponer un escenario futuro ideal - visión-, y se ideará y realizará un plan para alcanzarlo. A continuación, el modelo contempla la entrada en una espiral de mejora continua, repitiendo el proceso en planes bianuales. Es muy importante señalar que en los primeros estadios se debe "perder" todo el tiempo necesario en la sensibilización y creación de visiones compartidas y compromisos de actuación, sin los cuales el proyecto está destinado a sufrir problemas continuos que quizá conlleven su abandono.

\section{Notas}

1. Dentro del campo de la salud tenemos un ejemplo concreto del entronque entre la Medicina Basada en la Evidencia con las nuevas tecnologías al servicio de la clínica, la investigación y la gestión (Rodrigues, 2000).

2. El HGP está soportado por una magnífica infraestructura tecnológica que relaciona a toda la comunidad de expertos que trabajan en el mapeo y análisis funcional del genoma humano. A través de dicho sistema los investigadores tienen acceso a todas las bases de datos relacionadas con su trabajo: el GenBank (NCBI) de secuencias, las bases de datos de estructuras moleculares, y la base de datos de referencias bibliográficas MedLine. Además, dichas bases de datos están perfectamente integradas entre 
sí. Por último, el sistema publica la información en tiempo real e incluso incorpora mecanismos de filtrado de la información y de difusión selectiva.

3. Así por ejemplo, un reciente estudio (Brockopp et al., 1998) muestra como aspectos de cultura organizacional y carencias cognitivas del personal dificultan la implantación de programas de gestión del dolor de forma más decisiva que los factores meramente económicos. Los autores citan siete barreras principales: la carencia de conocimientos, las actitudes contrarias, liderazgos inconsistentes, relaciones laborales pobres, prejuicios culturales y religiosos, temores de los médicos ante las consecuencias legales y, solo en séptimo lugar, la falta de recursos.

\section{Agradecimientos}

El autor agradece la oportunidad de haber podido desarrollar durante una década la aplicación de la gestión del conocimiento al campo de la promoción de la salud a D. Javier Gallego Díez, Jefe de Sección de Educación de la Salud del Gobierno de Aragón, y a sus colaboradores. Agradece igualmente la oportunidad única de sintetizar sus ideas en el campo de la gestión del conocimiento que le proporcionaron D. Álvaro Quijano y el equipo directivo del Instituto Mexicano de Administración del Conocimiento al confiar en él para impartir dos cursos sobre el tema en el Colegio de México durante la primavera y el otoño de 2001. Finalmente, desea agradecer sus sugerencias a los revisores anónimos.

\section{Referencias}

Boguski, M. S. (1995). Adventures in Information Space: Biomedical Discoveries in a Molecular Sequence Milieu. // Serials librarian. 25:3-4 (1995), 125-131.

Brockopp, D. Y.; Brockopp, G.; Warden, S.; Wilson, J. A. (1998). Pain Management Project. // International Journal of Nursing Studies. 35:4); 226-232.

Broering, N. C. (1995). Changing Focus: Tomorrows Virtual Library. // Serials librarian. 25:3-4), 73-94.

Febrel Bordejé, Mercedes; Gallego, Javier; García Marco, Francisco Javier; Tejero Vela, María Cruz (1999). Desarrollo e integración de tesauros en promoción y educación para la salud. // Organización del Conocimiento en Sistemas de Información y Documentación. 3: Actas del III Encuentro de ISKO-España, Getafe, 19-21 de noviembre de 1997. Zaragoza: Universidad, 1999. 327-333.

García Marco, Francisco Javier (1999). La transferencia del saber de salud. // Actas de las III Jornadas Aragonesas de Educación para la Salud. Zaragoza: Universidad; Diputación General de Aragón; Ministerio de Educación y Cultura, 1999. 37-48.

García Marco, Francisco Javier (en edición). La administración del conocimiento: conceptos y aplicaciones. México, D. F.: Colegio de México, en edición.

Layton B.; Hahn, K. (1995). The Librarian as a Partner in Nursing-Education. // Bulletin of the Medical Library Association. 83:4 (1995) 499-502.

Matheson, N. W. (1995). Things to Come: Postmodern Digital Knowledge Management and Medical Informatics. // Journal of the american medical informatics association. 2:2 (1995) 73-78.

Scire. $9: 1$ (jul.-dic. 2003) 151-170. 
Nutley, S. M.; Davies, H. T. O. (2001). Developing Organizational Learning in the NHS. // Medical Education. 35:1 (2001) 35-42.

Rodrigues, R. J. (2000). Information-Systems The Key to Evidence-Based Health Practice. // Bulletin of the world health organization. 78:11 (2000) 1344-1351. 